\title{
Additive Schwarz preconditioners for a fully-discrete and symmetric boundary element method
}

\author{
Thanh Tran*
}

(Received 7 August 2000)

\begin{abstract}
We discuss various additive Schwarz preconditioners for a fullydiscrete and symmetric boundary element method when used to solve a Dirichlet problem in the plane. These preconditioners work in the

*Centre for Mathematics and its Applications, School of Mathematical Sciences, Australian National University, Canberra ACT 0200, Australia. mailto: Thanh. Tran@anu.edu. au

${ }^{0}$ See http: //anziamj. austms .org.au/V42/CTAC99/Tran for this article and ancillary services, (c) Austral. Mathematical Soc. 2000. Published 27 Nov 2000.
\end{abstract}


same way as when they are used for the Galerkin boundary element method: the condition numbers of the preconditioned stiffness matrices grow at most logarithmically with the degree of freedom. Several numerical results are presented to support the theory.

\section{Contents}

1 Introduction

C1422

2 The boundary-integral equation

C1423

3 The fully-discrete and symmetric method

C1425

4 Additive Schwarz preconditioners

C1428

4.1 General framework . . . . . . . . . . . . . . . . . . . C1428

4.2 Two-level methods . . . . . . . . . . . . . . . C1431

4.2.1 Non-overlapping method . . . . . . . . . . . . C C1432

4.2.2 Overlapping method ............. . . C1433

4.3 Multilevel methods . . . . . . . . . . . . . . . . C1435

5 Implementation

C1436

6 Numerical results

C1437

7 Conclusion

C1440 


\section{Introduction}

We consider in this paper a boundary-value problem for the Laplace equation in a bounded domain in $\mathrm{R}^{2}$ with smooth boundary. Via the standard fundamental solution, we reformulate the problem as a boundary-integral equation of the first kind with a symmetric kernel. A fully-discrete and symmetric method for this equation was suggested in [5], which results in solving a linear system the coefficient matrix $\mathcal{A}$ of which is dense. If $N$ is the size of $\mathcal{A}$, then the Gauss solver requires $O\left(N^{3}\right)$ operations for computation of the coefficients giving the solution of the fully-discrete method. Hence when $N$ is large one resorts to iterative methods, among which the conjugate gradient method is the most practical and efficient one. Since $\mathcal{A}$ is ill-conditioned, in the sense that its condition number increases with $N$, the convergence rate of the iterative method deteriorates, which leads to a large number of iterations needed. Preconditioners are necessary to reduce the cost of computation.

In this paper we shall discuss the use of additive Schwarz methods as preconditioners for the above linear system. Non-overlapping and overlapping two-level methods, and a multilevel method are discussed. We shall prove that the condition numbers of the preconditioned systems grow at most logarithmically with the degrees of freedom. 
For a fairly complete list of references to works on preconditioners for boundary-integral equations, the reader is referred to [8]. Multigrid analysis of finite element methods with numerical integration for partial differential equations has been discussed in $[1,3]$.

We should like to note that for the problem considered in this paper, due to the smoothness of the solution, a direct method may be sufficient in solving the linear system. However, this study is a step along the way to developing preconditioners for more demanding problems, where they are truly needed. Furthermore, the results in this paper apply not only to the logarithmic boundary integral equation but also to any equation of the form (6) in which the bilinear form $l(\cdot, \cdot)$ is equivalent to the $H^{-1 / 2}$ norm.

\section{The boundary-integral equation}

We consider the problem

$$
\begin{aligned}
\Delta U & =0 \quad \text { in } \Omega \\
U & =f \text { on } \Gamma,
\end{aligned}
$$

where $\Omega \subset \mathrm{R}^{2}$ is a bounded domain with smooth boundary $\Gamma$. Let $\nu_{\mathbf{y}}$ denote the outward unit normal at $\mathbf{y} \in \Gamma$. It is known that $U$ can be represented as

$$
U(\mathbf{x}):=\frac{1}{2 \pi} \int_{\Gamma} \phi(\mathbf{y}) \log \frac{\alpha}{|\mathbf{x}-\mathbf{y}|} d \sigma_{\mathbf{y}}-\frac{1}{2 \pi} \int_{\Gamma} f(\mathbf{y}) \frac{\partial}{\partial \nu_{\mathbf{y}}} \log \frac{1}{|\mathbf{x}-\mathbf{y}|} d \sigma_{\mathbf{y}}
$$


for $\mathbf{x} \in \Omega$, where $\phi$ is the normal derivative of $U$ with respect to $\nu$, and $\alpha$ is a positive parameter to be specified later. Taking the trace on $\Gamma$ and using the jump relations of the single- and double-layer potentials, we deduce that $\phi$ satisfies

$$
\frac{1}{2 \pi} \int_{\Gamma} \phi(\mathbf{y}) \log \frac{\alpha}{|\mathbf{x}-\mathbf{y}|} d \sigma_{\mathbf{y}}=F(\mathbf{x}), \quad \mathbf{x} \in \Gamma,
$$

where

$$
F(\mathbf{x}):=\frac{1}{2} f(\mathbf{x})+\frac{1}{2 \pi} \int_{\Gamma} f(\mathbf{y}) \frac{\partial}{\partial \nu_{\mathbf{y}}} \log \frac{1}{|\mathbf{x}-\mathbf{y}|} d \sigma_{\mathbf{y}}, \quad \mathbf{x} \in \Gamma .
$$

Let $\gamma$ be a 1-periodic parametric representation of $\Gamma$ such that $\gamma \in C^{\infty}$ and $\left|\gamma^{\prime}(x)\right| \neq 0$ for $x \in \mathrm{R}$ and let

$$
u(x):=\frac{1}{2 \pi} \phi[\gamma(x)]\left|\gamma^{\prime}(x)\right| \text { and } \quad g(x):=F[\gamma(x)] .
$$

Then we can recast (2) as

$$
\int_{0}^{1} K(x, y) u(y) d y=g(x), \quad 0 \leq x \leq 1,
$$

where

$$
K(x, y):=\log \frac{\alpha}{|\gamma(x)-\gamma(y)|} \quad \forall x, y \in[0,1]
$$

Let

$$
l(v, w):=\int_{0}^{1} \int_{0}^{1} K(x, y) v(x) w(y) d x d y
$$


We solve (3) in the weak form: find $u \in H^{-1 / 2}$ such that

$$
l(u, v)=\langle g, v\rangle \quad \forall v \in H^{-1 / 2} .
$$

Here, for all $s \in \mathrm{R}, H^{s}$ denotes the usual 1-periodic Sobolev space of order $s$ with norm denoted by $\|\cdot\|_{s}$.

The parameter $\alpha$ in the definition (4) of $K$ is chosen to satisfy

$$
\alpha \geq \operatorname{logarithmic} \text { capacity of } \Gamma \text {, }
$$

so that

$$
l(v, v) \geq \lambda\|v\|_{-1 / 2}^{2} \quad \forall v \in H^{-1 / 2} .
$$

For an explanation of the logarithmic capacity, see e.g. [4]. In practice, a simple way of guaranteeing that (7) holds is to choose $\alpha$ to be greater than the diameter of $\Gamma$.

\section{The fully-discrete and symmetric method}

In the standard Galerkin method, we seek a numerical solution $u_{h}^{*} \approx u$ by choosing a finite dimensional space $S_{h}$ and requiring that $u_{h}^{*} \in S_{h}$ satisfy

$$
l\left(u_{h}^{*}, v\right)=\langle g, v\rangle \quad \forall v \in S_{h} .
$$

Galerkin's method is a standard discretisation of (6), but in the system of linear equations arising from (9) the coefficients and right-hand sides involve 
complicated integrals that usually cannot be evaluated analytically. Hence, we are led to consider a fully-discrete Galerkin method in which the numerical solution $u_{h} \in S_{h}$ satisfies

$$
l_{h}\left(u_{h}, v\right)=\langle g, v\rangle_{h} \quad \forall v \in S_{h} .
$$

Here $l_{h}$ and $\langle\cdot, \cdot\rangle_{h}$ are certain discrete approximations to $l$ and $\langle\cdot, \cdot\rangle$. The forms $l_{h}$ and $\langle\cdot, \cdot\rangle_{h}$ are constructed in such a way that $u_{h}$ achieves the same rates of convergence as $u_{h}^{*}$ in the appropriate Sobolev norms. In [5] $l_{h}$ is designed to preserve the symmetry of the exact problem so that

$$
l_{h}(v, w)=l_{h}(w, v) \quad \forall v, w \in S_{h} .
$$

In the following we choose $S_{h}$ to be the space of piecewise-constant functions on a 1-periodic uniform mesh with $N$ subintervals between 0 and 1 defined by $t_{j}:=j h$ for $j=0, \ldots, N$, where $h:=1 / N$.

We follow $\left[5\right.$, Section 5] to define $l_{h}$ as follows. Let

$$
a=1 / 2, \quad b=0.1262814358793956, \quad c=0.6841827226000933, \quad d=1 / 6,
$$

or

$$
a=1 / 2, \quad b=0.4992036265530720, \quad c=0.0398933386986113, \quad d=1 / 6,
$$

and let $s_{1}=s_{6}=a, s_{2}=s_{5}=0, s_{3}=s_{4}=-a, \sigma_{1}=\sigma_{6}=b, \sigma_{2}=\sigma_{5}=c$, $\sigma_{3}=\sigma_{4}=b, w_{1}=w_{6}=d, w_{2}=w_{5}=1 / 2-2 d$, and $w_{3}=w_{4}=d$. With the 
quadrature weights being $\left\{w_{1}, \ldots, w_{6}\right\}$ and the quadrature points being

$$
\left(\xi_{p}, \eta_{p}\right):=\frac{1}{2}\left(1+s_{p}-\sigma_{p}, 1+s_{p}+\sigma_{p}\right), \quad p=1, \ldots, 6,
$$

we define

$$
l_{h}(v, w):=h^{2} \sum_{j=0}^{N-1} \sum_{k=0}^{N-1} \sum_{p=1}^{6} w_{p} K\left(x_{j p}, y_{k p}\right) v\left(y_{k p}\right) w\left(x_{j p}\right),
$$

where $x_{j p}:=t_{j}+\xi_{p} h$ and $y_{k p}:=t_{k}+\eta_{p} h$. We note that the quadrature points $\left(\xi_{p}, \eta_{p}\right), p=1, \ldots, 6$, are symmetric about both diagonals of the original unit square.

For the discrete inner product $\langle\cdot, \cdot\rangle_{h}$ we also choose the quadrature proposed in [5, Section 5] which is a 2-point Gauss-Legendre rule of degree of precision 2 with quadrature points $\xi_{1}^{\prime}=0.5(1-1 / \sqrt{3}), \xi_{2}^{\prime}=0.5(1+1 / \sqrt{3})$, and weights $w_{1}^{\prime}=w_{2}^{\prime}=0.5$. We then define

$$
\langle v, w\rangle_{h}:=h \sum_{j=0}^{N-1} \sum_{p=1}^{2} w_{p}^{\prime} v\left(x_{j p}^{\prime}\right) w\left(x_{j p}^{\prime}\right),
$$

where $x_{j p}^{\prime}:=t_{j}+\xi_{p}^{\prime} h$.

With $l_{h}$ and $\langle\cdot, \cdot\rangle_{h}$ defined as above, it follows from [5, Theorem 5.1] that for $h$ sufficiently small there exists a unique solution $u_{h} \in S_{h}$ of (10) satisfying

$$
\left\|u_{h}-u\right\|_{s} \leq c h^{t-s}\|u\|_{t+\max (-1-s, 0)}
$$


for $s<1 / 2,-1 / 2<t$, and $-1 \leq s \leq t \leq 1$. Moreover, the discrete Galerkin matrix is positive definite.

We note that the method designed as above is of translation-invariant order 3 and that the orders of precision of the left-hand side and right-hand side are both at least 3 ; see [5, Definition 4.2 and page 337].

\section{Additive Schwarz preconditioners}

\subsection{General framework}

In this subsection, we present the additive Schwarz method in an abstract form. We consider a general problem on a finite-dimensional space $\mathcal{S}$

$$
\text { Find } u \in \mathcal{S} \text { such that } L(u, v)=G(v) \quad \forall v \in \mathcal{S},
$$

where $L(\cdot, \cdot): \mathcal{S} \times \mathcal{S} \longrightarrow \mathrm{R}$ is a symmetric, bounded, and coercive bilinear form, and $G: \mathcal{S} \longrightarrow \mathrm{R}$ is a bounded linear functional.

To define an additive Schwarz method for (11), we represent $\mathcal{S}$ as

$$
\mathcal{S}=\mathcal{S}_{0}+\cdots+\mathcal{S}_{J}
$$

where $\mathcal{S}_{i}, i=0, \ldots, J$, are subspaces of $\mathcal{S}$. Let $P_{i}: \mathcal{S} \longrightarrow \mathcal{S}_{i}$ be the projection defined by

$$
L\left(P_{i} v, w\right)=L(v, w) \quad \forall v \in \mathcal{S}, w \in \mathcal{S}_{i} .
$$


Denoting

$$
P:=\sum_{i=0}^{J} P_{i}
$$

we solve the variational problem (11) by solving

$$
P u=\tilde{G},
$$

where $\tilde{G}:=\sum_{i=0}^{J} G_{i}$ with $G_{i} \in \mathcal{S}_{i}$ being a solution of

$$
L\left(G_{i}, w\right)=G(w) \quad \forall w \in \mathcal{S}_{i} .
$$

The $l_{2}$ condition number of $P$ is given by $\kappa(P)=\lambda_{\max }(P) / \lambda_{\min }(P)$ where the extremum eigenvalues are related to the decomposition (12) in the following manner, see e.g. [10],

$$
\lambda_{\min }(P)=\min _{v \in \mathcal{S}} \frac{L(v, v)}{\min _{\sum v_{i}=v} \sum_{i} L\left(v_{i}, v_{i}\right)} \quad \text { and } \quad \lambda_{\max }(P)=\max _{v \in \mathcal{S}} \frac{L(v, v)}{\min _{\sum v_{i}=v} \sum_{i} L\left(v_{i}, v_{i}\right)}
$$

Here $v=\sum_{i} v_{i}$ is a decomposition of $v$ with $v_{i} \in \mathcal{S}_{i}$. This decomposition may not be unique. It results from (16) that, see e.g. [10],

$$
\kappa(P) \leq \frac{\Lambda_{2}}{\Lambda_{1}}
$$

if the following two conditions are satisfied: 
(C1) For any $v \in \mathcal{S}$ and for any decomposition $v=\sum_{i} v_{i}, v_{i} \in \mathcal{S}_{i}$, there holds

$$
L(v, v) \leq \Lambda_{2} \sum_{i=0}^{J} L\left(v_{i}, v_{i}\right)
$$

(C2) For any $v \in \mathcal{S}$ there exists a decomposition $v=\sum_{i} v_{i}, v_{i} \in \mathcal{S}_{i}$, such that

$$
\Lambda_{1} \sum_{i=0}^{J} L\left(v_{i}, v_{i}\right) \leq L(v, v) .
$$

Our aim is to design additive Schwarz methods so that $\Lambda_{2} / \Lambda_{1}$ is bounded independently of (or grows at most logarithmically with) the degree of freedom. For the Galerkin approximation (9), it has been noted that the use of some extra preconditioner is necessary before applying additive Schwarz or multigrid algorithms. In [2], a difference quotient was used, whereas the use of Haar bases was mentioned in $[6,7,8,9]$. In this paper, Haar bases will be used in the design of additive Schwarz methods for the fully-discrete approximation (10).

Before moving to the description of the methods, we prove the following lemma which yields the equivalence of the two bilinear forms $l$ and $l_{h}$.

Lemma 1 There exists positive constants $C_{1}$ and $C_{2}$ such that

$$
C_{1} l(v, v) \leq l_{h}(v, v) \leq C_{2} l(v, v) \quad \forall v \in S_{h}
$$


Proof: The boundedness of the form $l(\cdot, \cdot)$ and Theorem 2.1 (i) and Corollary 3.9 in [5] immediately give the left-hand inequality. To prove the righthand inequality we first note that Lemmas 4.4 and 3.5 in [5] yield, respectively (noting Theorem 2.1 (v) in the same paper),

$$
\left|a_{h}(v, v)\right| \leq|a(v, v)|+c\|v\|_{-1 / 2}^{2} \leq c\|v\|_{-1 / 2}^{2} \quad \forall v \in S_{h},
$$

and

$$
\left|b_{h}(v, v)\right| \leq|b(v, v)|+c\|v\|_{-1 / 2}^{2} \quad \forall v \in S_{h},
$$

where $a_{h}(\cdot, \cdot)$ and $b_{h}(\cdot, \cdot)$ are defined as in [5] so that

$$
l_{h}(v, w)=a_{h}(v, w)+b_{h}(v, w) \quad \forall v, w \in S_{h} .
$$

(Here and in the sequel $c$ denotes a generic positive constant which may take different values at different occurrences.) Therefore by using (8) we obtain

$$
\begin{aligned}
l_{h}(v, v) & \leq\left|a_{h}(v, v)\right|+\left|b_{h}(v, v)\right| \leq c\|v\|_{-1 / 2}^{2}+|b(v, v)| \\
& \leq c\|v\|_{-1 / 2}^{2}+l(v, v) \leq c l(v, v) \quad \forall v \in S_{h} .
\end{aligned}
$$

The lemma is proved.

\subsection{Two-level methods}

The convergence result for the fully-discrete method (10) discussed in [5] being established under the assumption that the meshes are uniform, we assume in the following uniformity of a two-level mesh to simplify our presentation, even though quasi-uniformity is allowed for the Galerkin method. 
The coarse mesh: We first divide $I$ into disjoint subdomains $I_{i}$ with length $H, i=1, \ldots, J$, so that $\bar{I}=\cup_{i=1}^{J} \bar{I}_{i}$.

The fine mesh: Each $I_{i}$ is further divided into disjoint subintervals $I_{i j}$ with length $h, j=1, \ldots, N$, so that $\bar{I}_{i}=\cup_{j=1}^{N} \bar{I}_{i j}$.

We recall that the finite-dimensional space $\mathcal{S}=S_{h}$ is defined as the space of 1-periodic piecewise-constant functions on the fine mesh. For both non-overlapping and overlapping methods, we define $\mathcal{S}_{0}$ to be the space of 1 -periodic piecewise-constant functions on the coarse mesh.

The subspaces $\mathcal{S}_{i}, i=1, \ldots, J$, will be defined as spaces of functions which are derivatives of piecewise-linear functions. We denote by $\mathcal{T}$ the space of piecewise-linear functions on the fine mesh.

\subsubsection{Non-overlapping method}

Let

$$
\mathcal{T}_{i}:=\left\{v \in \mathcal{T} \mid \operatorname{supp} v \subset \bar{I}_{i}\right\}, \quad i=1, \ldots, J,
$$

and let

$$
\mathcal{S}_{i}:=\left\{w \mid \exists v \in \mathcal{T}_{i}: w=v^{\prime}\right\}, \quad i=1, \ldots, J .
$$

Bases for $\mathcal{S}_{i}$ can be formed by the Haar functions; see [9]. 
The decomposition of $\mathcal{S}$ by (12) with $\mathcal{S}_{i}$ defined as above allows us to use (13) and (14) to define additive Schwarz operators $P_{G}$ and $P_{F}$ when $L=l$ (Galerkin approximation, see (11)) and when $L=l_{h}$ (fully-discrete method), respectively.

Theorem 2 The condition number of the non-overlapping additive Schwarz operator $P_{F}$ is bounded as

$$
\kappa\left(P_{F}\right)=O\left(1+\log \frac{H}{h}\right) .
$$

Proof: Lemmas 3.4 and 3.7 in [6] assure us that conditions (C1) and (C2) hold for $L=l$ with

$$
\Lambda_{1}=c\left(1+\log \frac{H}{h}\right)^{-1},
$$

and $\Lambda_{2}$ being a constant independent of $H$ and $h$. In view of Lemma 1, the same results hold for $L=l_{h}$ (with different constants), implying (19); see (17).

\subsubsection{Overlapping method}

We now extend each subdomain $I_{i}$ on each side by a fixed number of subintervals so that the length of the overlap between two extended subdomains 
$I_{i}^{\prime}$ and $I_{i+1}^{\prime}$ is $2 \delta$ for some $\delta \in(0, H]$ which is a multiple of $h$. This implies

$$
\left|I_{i}^{\prime}\right| \sim H .
$$

The subspaces $\mathcal{S}_{i}, i=1, \ldots, J$, are now defined as in (18) with $\mathcal{T}_{i}$ now defined by

$$
\mathcal{T}_{i}:=\left\{v \in \mathcal{T} \mid \operatorname{supp} v \subset \bar{I}_{i}^{\prime}\right\}, \quad i=1, \ldots, J .
$$

This overlapping decomposition completely defines the additive Schwarz operators $P_{G}$ and $P_{F}$.

Theorem 3 The condition number of the overlapping additive Schwarz operator $P_{F}$ is bounded as

$$
\kappa\left(P_{F}\right)=O\left(1+\log ^{2} \frac{H}{\delta}\right) .
$$

Proof: Following the argument in the proof of Lemma 3.4 in [6] and using Lemma 5.2 of [8] in lieu of [6, Eqn (3.17)] we obtain (C2) for $L=l$ with

$$
\Lambda_{1}=c\left(1+\log ^{2} \frac{H}{\delta}\right)^{-1} .
$$

The constant $\Lambda_{2}$ in (C1) can also be obtained from Lemma 5.1 in [8], using the same approach. Thus Lemma 1 yields the desired result. 


\subsection{Multilevel methods}

The multilevel method is defined as follows. Starting with a coarse mesh

$$
\mathcal{N}^{1}: 0=x_{0}^{1}<x_{1}^{1}=1 / 2<x_{2}^{1}=1,
$$

we divide each subinterval into two equal intervals. Hence, if $h_{l}$ is the meshstep of $\mathcal{N}^{l}, l=1, \ldots, L-1$, then $h_{l}=2 h_{l+1}$. For $l=1, \ldots, L$ and $i=0, \ldots, N_{l}:=2^{l}$, let $\phi_{i}^{l}$ be the hat function which takes the value 1 at $x_{i}^{l}$ and zero at the other mesh points. We then define $\mathcal{S}_{i}^{l}:=\operatorname{span}\left\{\psi_{i}^{l}\right\}$, where the Haar basis function $\psi_{i}^{l}$ is the derivative of $\phi_{i}^{l}$. The space $\mathcal{S}$ is now decomposed by

$$
\mathcal{S}=\mathcal{S}_{0}+\sum_{l=2}^{L} \sum_{i=1}^{N_{l}} \mathcal{S}_{i}^{l} .
$$

It was proved in [9] that the condition number of the multilevel additive Schwarz operator $P_{G}$ is bounded independently of the number of level $L$ and the number of mesh points. Using Lemma 1 again we deduce

Theorem 4 The condition number of the multilevel additive Schwarz operator $P_{F}$ is bounded independently of the number of level $L$ and the number of mesh points. 


\section{Implementation}

We briefly discuss in this section the implementation aspect of the two-level methods. For the implementation of the multilevel method the reader is referred to e.g. $[9,10]$.

Let $\phi_{j}$ be the brick function in the interval $\left(t_{j-1}, t_{j}\right)$ and let $\mathbf{A}_{k l}, k, l=$ $1, \ldots, J$, be sub-blocks of the stiffness matrix $\mathbf{A}$ arising from (10) defined by $\mathbf{A}_{k l}:=\left(a_{i j}^{k l}\right)$ with $a_{i j}^{k l}=l_{h}\left(\phi_{i}, \phi_{j}\right)$. Here $\phi_{i}$ and $\phi_{j}$ are brick functions such that supp $\phi_{i} \subset \bar{I}_{k}$ and supp $\phi_{j} \subset \bar{I}_{l}$ for the non-overlapping method, and supp $\phi_{i} \subset \bar{I}_{k}^{\prime}$ and supp $\phi_{j} \subset \bar{I}_{l}^{\prime}$ for the overlapping method. Then $\mathbf{A}$ has the form

$$
\left(\begin{array}{cccc}
\mathbf{A}_{11} & \mathbf{A}_{12} & \ldots & \mathbf{A}_{1 J} \\
& \mathbf{A}_{22} & \ldots & \mathbf{A}_{2 J} \\
& & \ddots & \vdots \\
& & & \mathbf{A}_{J J}
\end{array}\right) .
$$

The matrix representation $\mathbf{B}$ of the non-overlapping preconditioner will now have the form

$$
\left(\begin{array}{cccc}
\mathbf{A}_{11}^{-1} & \mathbf{O} & \cdots & \mathbf{O} \\
& \mathbf{A}_{22}^{-1} & \cdots & \mathbf{O} \\
& & \ddots & \vdots \\
& & & \mathbf{A}_{J J}^{-1}
\end{array}\right)+\Pi \mathbf{A}_{0}^{-1} \Pi^{t},
$$

where $\mathbf{A}_{0}$ is the stiffness matrix on the coarse mesh level, $\Pi$ is the interpolation operator from the coarse mesh level to the fine mesh level, and 
$\Pi^{t}$ is its adjoint operator. Let $\mathbf{v}$ be the vector representation of a function $v=\sum_{i=0}^{J} v_{i} \in \mathcal{S}, v_{i} \in \mathcal{S}_{i}$. To compute $\mathbf{B v}$, there is no need to know the explicit form of $\mathbf{B}$. It suffices to solve the local problems $\mathbf{A}_{i i} \mathbf{w}_{i}=\mathbf{v}_{i}$, $i=1, \ldots, J$, and the global problem $\mathbf{A}_{0} \mathbf{w}_{0}=\Pi^{t} \mathbf{v}_{0}$, and obtain $\mathbf{B v}$ as

$$
\mathbf{B v}=\Pi \mathbf{w}_{0}+\sum_{i=1}^{J} \mathbf{w}_{i} .
$$

However, before doing so one has to transform the representation of the function $v$ in the usual basis to the Haar basis; see Section 4. After obtaining the resulting function from $\mathbf{B v}$, a reverse transform to the usual basis is necessary.

\section{$6 \quad$ Numerical results}

In our numerical experiments we considered the problem (1) for $\Gamma$ being the ellipse with semi-axes $a_{1}=4$ and $a_{2}=2$. The logarithmic capacity of $\Gamma$ in this case is $\left(a_{1}+a_{2}\right) / 2=3$. In our numerical calculations we chose $\alpha=3.5$. The boundary condition was chosen to be $f(\mathbf{x})=\sqrt{\left|x_{1}+x_{2}^{2}\right|}$, where $\mathbf{x}=\left(x_{1}, x_{2}\right)$.

We solved the problem using the fully-discrete method described in Section 3. The linear systems were solved via the conjugate gradient method, with and without preconditioning. The iterations were stopped when the 
TABLE 1: (1): unpreconditioned, (2): preconditioned by non-overlapping method, (3): preconditioned by overlapping method, (4): preconditioned by multilevel method

\begin{tabular}{|c|c|c|c|c|c|c|c|c|}
\hline & \multicolumn{5}{|c|}{ Condition numbers } & \multicolumn{4}{|c|}{ Iterations } \\
\hline DoF & $(1)$ & $(2)$ & $(3)$ & $(4)$ & $(1)$ & $(2)$ & $(3)$ & $(4)$ \\
\hline 4 & $0.366 \mathrm{E}+01$ & $0.366 \mathrm{E}+01$ & $0.366 \mathrm{E}+01$ & $0.234 \mathrm{E}+01$ & 2 & 2 & 2 & 2 \\
8 & $0.474 \mathrm{E}+01$ & $0.474 \mathrm{E}+01$ & $0.474 \mathrm{E}+01$ & $0.457 \mathrm{E}+01$ & 4 & 4 & 4 & 4 \\
16 & $0.116 \mathrm{E}+02$ & $0.193 \mathrm{E}+02$ & $0.474 \mathrm{E}+01$ & $0.665 \mathrm{E}+01$ & 7 & 8 & 8 & 7 \\
32 & $0.242 \mathrm{E}+02$ & $0.253 \mathrm{E}+02$ & $0.315 \mathrm{E}+01$ & $0.290 \mathrm{E}+01$ & 15 & 12 & 11 & 10 \\
64 & $0.489 \mathrm{E}+02$ & $0.319 \mathrm{E}+02$ & $0.281 \mathrm{E}+01$ & $0.105 \mathrm{E}+02$ & 24 & 14 & 10 & 13 \\
128 & $0.977 \mathrm{E}+02$ & $0.389 \mathrm{E}+02$ & $0.105 \mathrm{E}+02$ & $0.122 \mathrm{E}+02$ & 34 & 15 & 12 & 13 \\
256 & $0.195 \mathrm{E}+03$ & $0.467 \mathrm{E}+02$ & $0.115 \mathrm{E}+02$ & $0.144 \mathrm{E}+02$ & 47 & 16 & 13 & 14 \\
512 & $0.390 \mathrm{E}+03$ & $0.550 \mathrm{E}+02$ & $0.128 \mathrm{E}+02$ & $0.164 \mathrm{E}+02$ & 64 & 17 & 13 & 14 \\
1024 & $0.779 \mathrm{E}+03$ & $0.641 \mathrm{E}+02$ & $0.145 \mathrm{E}+02$ & $0.183 \mathrm{E}+02$ & 87 & 18 & 14 & 14 \\
\hline
\end{tabular}


TABLE 2: (1): unpreconditioned, (2): preconditioned by non-overlapping method, (3): preconditioned by overlapping method, (4): preconditioned by multilevel method

\begin{tabular}{|c|c|c|c|c|}
\hline & \multicolumn{4}{|c|}{ CPU times } \\
\hline DoF & $(1)$ & $(2)$ & $(3)$ & $(4)$ \\
\hline 4 & $0.635 \mathrm{E}-01$ & $0.704 \mathrm{E}-03$ & $0.669 \mathrm{E}-03$ & $0.648 \mathrm{E}-01$ \\
8 & $0.106 \mathrm{E}-02$ & $0.922 \mathrm{E}-03$ & $0.980 \mathrm{E}-03$ & $0.468 \mathrm{E}-02$ \\
16 & $0.153 \mathrm{E}-02$ & $0.201 \mathrm{E}-01$ & $0.219 \mathrm{E}-01$ & $0.106 \mathrm{E}-01$ \\
32 & $0.410 \mathrm{E}-02$ & $0.343 \mathrm{E}-01$ & $0.371 \mathrm{E}-01$ & $0.206 \mathrm{E}-01$ \\
64 & $0.142 \mathrm{E}-01$ & $0.615 \mathrm{E}-01$ & $0.536 \mathrm{E}-01$ & $0.396 \mathrm{E}-01$ \\
128 & $0.572 \mathrm{E}-01$ & $0.149 \mathrm{E}+00$ & $0.169 \mathrm{E}+00$ & $0.695 \mathrm{E}-01$ \\
256 & $0.594 \mathrm{E}+00$ & $0.608 \mathrm{E}+00$ & $0.533 \mathrm{E}+00$ & $0.264 \mathrm{E}+00$ \\
512 & $0.719 \mathrm{E}+01$ & $0.359 \mathrm{E}+01$ & $0.281 \mathrm{E}+01$ & $0.165 \mathrm{E}+01$ \\
1024 & $0.520 \mathrm{E}+02$ & $0.188 \mathrm{E}+02$ & $0.150 \mathrm{E}+02$ & $0.878 \mathrm{E}+01$ \\
\hline
\end{tabular}


$l_{2}$ norm of the residual was less than $10^{-9}$, and all calculations were performed in double precision. The Lanczos algorithm was used to compute the condition numbers.

Table 1 shows the condition numbers and numbers of iterations for the unpreconditioned systems and the systems preconditioned by the non overlapping, overlapping, and multilevel methods designed in Section 4. Table 2 shows the cpu times for each of the methods.

\section{Conclusion}

We designed in this paper a non-overlapping and an overlapping two-level method, and a multilevel method for the linear system arising from a fullydiscrete and symmetric boundary element method used to solve a Dirichlet problem. The preconditioners work in the same way as in the case of the Galerkin method: the condition number grows at most logarithmically with the degree of freedom. 


\section{References}

[1] J. H. Bramble, C. I. Goldstein, and J. E. Pasciak. Analysis of V-cycle multigrid algorithms for forms defined by numerical quadrature. SIAM J. Sci. Comput., 15:566-576, 1994. C1423

[2] J. H. Bramble, Z. Leyk, and J. E. Pasciak. The analysis of multigrid algorithms for pseudodifferential operators of order minus one. Math. Comp., 63:461-478, 1994. C1430

[3] C. I. Goldstein. Multigrid analysis of finite element methods with numerical integration. Math. Comp., 56:409-436, 1991. C1423

[4] E. Hille. Analytic Function Theory, volume II. Ginn, Boston, 1962. C1425

[5] W. McLean and I. H. Sloan. A fully discrete and symmetric boundary element method. IMA J. Numer. Anal., 14:311-345, 1994. C1422, C1426, C1426, C1427, C1427, C1428, C1431, C1431, C1431, C1431

[6] E.P. Stephan and T. Tran. Domain decomposition algorithms for indefinite weakly singular integral equations: the $h$ and $p$ versions. IMA J. Numer. Anal., 20:1-24, 2000. C1430, C1433, C1434, C1434

[7] T. Tran. Additive Schwarz algorithms and the Galerkin boundary element method. In J. Noye, M. Teubner, and A. Gill, editors, 
Computational Techniques and Applications: CTAC97, pages 703-710, Singapore, 1998. World Scientific. C1430

[8] T. Tran. Overlapping additive Schwarz preconditioners for boundary element methods. J. Integral Eqns Appl., to appear. C1423, C1430, C1434, C1434

[9] T. Tran and E.P. Stephan. Additive Schwarz methods for the $h$ version boundary element method. Appl. Anal., 60:63-84, 1996. C1430, C1432, C1435, C1436

[10] X. Zhang. Multilevel Schwarz methods. Numer. Math., 63:521-539, 1992. C1429, C1429, C1436 